\title{
Characterization of group B coxsackieviruses isolated from non-polio acute flaccid paralysis patients in Pakistan: vital assessment before polio eradication
}

\author{
M. ANGEZ ${ }^{1,2}$, S. SHAUKAT ${ }^{1}$, R. ZAHRA ${ }^{2}$, M. M. ALAM ${ }^{1}$, S. SHARIF $^{1}$, \\ A. KHURSHID ${ }^{1}$, Y. ARSHAD ${ }^{1}$, M. SULEMAN ${ }^{1}$, G. MUJTABA ${ }^{1}$ AND \\ S. S. Z. ZAIDI ${ }^{1}$ \\ ${ }^{1}$ Department of Virology, National Institute of Health, Chak Shahzad, Park Road, Islamabad-45500, Pakistan \\ ${ }^{2}$ Department of Microbiology, Faculty of Biological Sciences, Quaid-i-Azam University, Islamabad-45320, \\ Pakistan
}

Received 27 February 2016; Final revision 23 June 2017; Accepted 27 June 2017; first published online 25 July 2017

\section{Summary}

Pakistan is at the verge of polio eradication but isolation of non-polio enteroviruses (NPEVs) from acute flaccid paralysis (AFP) cases may result in serious or even fatal outcome. Many enteroviruses share similar symptoms and epidemiology as is the case with poliovirus and coxsackievirus $(\mathrm{CV})$. The present study was designed to genetically characterize coxsackievirus B (CV-B) serotypes isolated from non-polio acute flaccid paralytic children, as well as to understand their probable role in paralysis. A total of $63(20 \cdot 1 \%)$ out of 313 stool samples during 2013 were found positive for NPEVs in rhabdomyosarcoma cells. Only $24(38.0 \%)$ NPEVs were typed as CV-B by microneutralization assay and were further characterized by sequencing of the viral protein 1 (VP1) gene. Molecular phylogenetic analyses classified the study strains into six coxsackievirus B serotypes (coxsackievirus B1 to B6) with their respective prototype strains with evidence of epidemiological linkage and distinct clusters. Moreover, four major differences were found within the amino acid sequences of BC-loop in VP1 of CV-B strains. In conclusion, this study presented the molecular evolutionary genetic overview and distinct phylogenetic pattern of CV-B isolates from AFP cases in Pakistan, and explored the possible link between CV-B infections and AFP cases. Furthermore, our data reveal that these viruses might contribute to the incidence of paralysis in population and there is need of time to establish an enterovirus surveillance system for better understanding of epidemiological and virological characteristics of NPEV infections associated with AFP cases in the country.

Key words: Acute flaccid paralysis, coxsackievirus B, enterovirus, molecular epidemiology, Pakistan.

\section{INTRODUCTION}

Coxsackievirus (CV) is a member of species Enterovirus B of genus Enterovirus in the family

\footnotetext{
* Author for correspondence: S. Shaukat, Department of Virology, National Institute of Health, Chak Shahzad, Park Road, Islamabad-45500, Pakistan.

(Email: vibgyors@yahoo.com)
}

Picornaviridae [1]. Originally, these viruses were discovered in 1948 by Gilbert Dalldorf from the fecal specimens of children with poliomyelitis like illness, residing in town 'Coxsackie' situated on the Hudson River in New York [2]. These are nonenveloped, single-stranded, positive sense RNA viruses of 7-8 $\mathrm{kb}$ pairs long and were divided into two subgroups called A and B based on characteristic disease induced in suckling mice inoculated intracerebrally. 
Coxsackievirus A (CV-A) cause a flaccid paralysis by infecting skeletal muscle, whereas coxsackievirus B (CV-B) cause a spastic paralysis due to focal muscle injury and degeneration of neuronal tissue [3]. Currently, $23 \mathrm{CV}-\mathrm{A}$ and six CV-B serotypes are recognized [4].

CV-B have worldwide distribution with different geographic patterns of circulation. These viruses have long been recognized as significant pathogen of infants and children and are also known as pediatric pathogen [5]. CV-B infections can result in various diseases, including aseptic meningitis, acute myocarditis and pericarditis, but most commonly recognized serious manifestation of CV-B infection is associated with paralysis alone or in combination with encephalitis. The presentation may mimic that of poliovirus related paralysis with fever at the time of onset of paralysis and a rapid progression to flaccid paralysis [6]. Nearly all serotypes of CV-B have been associated with paralytic infections [7]. These viruses can be isolated from CSF (cerebrospinal fluid), stool, rectal swabs, throat swabs and nasal secretions, but the isolation from stool is most sensitive due to the presence of virus in higher titer and longer than in any other specimen [8].

The application of molecular biology techniques to clinical virology has begun to significantly change approaches to enterovirus diagnostics. The sequence analysis of viral protein 1 (VP1; one of the main exposed regions of the viral capsid) has been successfully implemented for enterovirus serotype identification [9]. Currently, the evolutionary distances and phylogenetic investigations based on VP1 gene are used for the identification and classification of CV-B [9]. In addition, phylogenetic analyses based on sequencing data of VP1 region is considered to be the standard method of molecular analysis for epidemiological purpose or clustering or genotyping or to identify emerging new variants [10].

The objective of this study was to understand the molecular epidemiology of group B CVs isolated from the stool of non-polio acute flaccid paralytic children and to identify their probable role in causing paralysis from the perspective of poliomyelitis eradication.

\section{METHODS}

\section{Ethics statement}

This study was approved by Institutional Internal Review Committee of the National Institute of Health, Pakistan. Written informed consent was obtained from all the individuals participated. A check box was included in data form to document the consent taking procedure.

\section{Sample collection}

Stool samples ( $n=313$ ) were collected during 2013 from patients with acute flaccid paralysis (AFP) under the age of 15 years. All stool samples collected according to World Health Organization (WHO) guidelines within 14 days from the date of onset and were delivered to WHO Regional Reference Laboratory for Polio Eradication Initiative, Virology Department, National Institute of Health Islamabad, Pakistan and stored at $-20^{\circ} \mathrm{C}$.

\section{Virus isolation and microneutralization}

All these samples were processed as per WHO standard procedures [11]. Briefly, these samples were processed and inoculated into RD (human rhabdomyosarcoma) and L20B (recombinant murine cell lines expressing human poliovirus receptor) cells and were observed daily for the development of a characteristic enterovirus cytopathic effect (CPE). Isolates from RD cells were re-passaged to L20B cells, and were designated as nonpolio enterovirus (NPEV) if no CPE was observed. According to WHO standard protocols, typing of the NPEV isolates was carried out by microneutralization using typing sera kit [provided by National Institute for Public Health and the Environment (RIVM), Bilthoven] containing the polyclonal antisera to identify 23 enterovirus serotypes [11].

\section{VP1 amplification}

RNA was extracted from $140 \mu$ l of the infected cell culture using QIAamp viral RNA extraction kit (Qiagen, Valencia, CA, USA). NPEVs were confirmed by realtime reverse transcription polymerase chain reaction (qRT-PCR) targeting the $5^{\prime}$ untranslated region [12]. Reverse transcription-polymerase chain reaction (RTPCR) was performed to amplify the VP1 gene of NPEVs. Initially, complementary DNA (cDNA) was synthesized in a $20 \mu \mathrm{l}$ reaction mixture comprising of $11 \mu \mathrm{l}$ of each viral RNA, $4 \mu \mathrm{l}$ of $5 \times$ transcriptase buffer, $2 \mu \mathrm{l}$ of $10 \mathrm{mM}$ dithiothreitol (DTT), $0.5 \mu \mathrm{l}$ of $25 \mathrm{mM}$ each deoxynucleotide (dNTP) solution mix (Roche), 1 $\mu \mathrm{l}$ of $40 \mathrm{U}$ of ribonuclease Inhibitor, $1 \mu \mathrm{l}$ of $20 \mathrm{U}$ Avian Myeloblastosis Virus (AMV) reverse transcriptase (Thermo Scientific) and $0 \cdot 5 \mu \mathrm{l}$ of nuclease free 
water at $42{ }^{\circ} \mathrm{C}$ for $45 \mathrm{~min}$. For amplification of VP1 region RCR was performed by using $2 \mu$ of cDNA, $5 \mu \mathrm{l}$ of $10 \times \mathrm{Taq}$ buffer, $3.5 \mu \mathrm{l}$ of $\mathrm{MgCl}_{2}(50 \mathrm{mM}), 1 \cdot 0$ $\mu 1$ of $12.5 \mathrm{mM}$ dNTPs, $0.5 \mu \mathrm{l}$ of Taq DNA polymerase ( 5 units $/ \mu 1$ ), $37 \mu \mathrm{l}$ of water and $0.5 \mu \mathrm{l}$ of each primer (10 $\mu \mathrm{M}) 224$ and 222 targeting VP3 (nt 2204-2223) and VP1 (nt 2969-2951) region of virus genome, respectively [13]. Thermocycler parameters for RT-PCR was $95^{\circ} \mathrm{C}$ for $3 \mathrm{~min}$ followed by 40 cycles of $94^{\circ} \mathrm{C}$ for $45 \mathrm{~s}, 42^{\circ} \mathrm{C}$ for $45 \mathrm{~s}$ and $72{ }^{\circ} \mathrm{C}$ for $1 \mathrm{~min}$. Specific RT-PCR products were purified using the Qiaquick PCR purification kit (Qiagen) and stored at $-20^{\circ} \mathrm{C}$ till further procedure.

\section{Nucleotide sequencing and phylogenetic analyses}

The purified products were sequenced using the same forward and reverse primers as used in RT-PCR with the BigDye Terminator v3.1 cycle sequencing kit (Applied Biosystems, Foster City, CA), and sequencing was performed with ABI 3130 genetic analyzer (Applied Biosystems). The obtained sequences were curated and aligned using Sequencher version 4.9 software (GeneCodes) (http://www.genecodes.com). Molecular typing based on VP1 sequences was performed using automated genotyping tool for enterovirus version $0 \cdot 1$ [14]. VP1 sequences were also subjected to online BLAST analyses and the sequence of CV-B prototype and all the closest relative strains of study isolates retrieved from GenBank (http://www.ncbi.nlm.nih.gov/ BLAST). These sequences were aligned using ClustalW. The genetic distances were calculated by using Kimura-2 parameter model and Jones-ThorntonTaylor (JTT) model for nucleotide and deduced amino acid sequences, respectively. Phylogenetic tree was constructed using the neighbor-joining method with 1000 bootstrap replicates in MEGA software (version 5.0) [15]. Geneious Package (version 10·1·3; http://www.geneious.com) was used for VP1 deduced amino acid sequence analysis and to generate sequence logos, with columns of amino acids for each position in the sequence. The column height signifies conservation of the sequence at that position, while the height of the amino acids within the column shows relative frequency $[16,17]$.

The sequence data described in this study have been submitted to GenBank data library under accession numbers KY593460-KY593483.

\section{RESULTS}

A total of 313 stool samples from AFP patients were processed and inoculated on RD and L20B cell lines. Overall on cell culture, $63(20 \cdot 1 \%)$ stool samples were found positive for NPEV, $110(35 \cdot 1 \%)$ for poliovirus and 140 $(44 \cdot 7 \%)$ remained negative. Microneutralization assay successfully typed $56(88.8 \%)$ NPEV isolates into echovirus $(n=25 ; 44 \cdot 7 \%)$, numbered enterovirus $(n=7$; $12 \cdot 5 \%)$ and CV-B $(n=24 ; 42 \cdot 8 \%)$, while seven $(11 \cdot 1 \%)$ isolates remained untypable. All CV-B isolates were subjected to RT-PCR and characterized by VP1 gene sequencing. The partial (479 bp) VP1 nucleotide sequences of these isolates were compared with the CV-B sequences selected among those available in GenBank. The prototype sequence of each CV-B serotype was also included. BLAST analysis of the sequences indicated homology of the study strains to CV-B1 $(n=3)$, CV-B2 $(n=1)$, CV-B3 $(n=2)$, CV-B4 $(n=1)$, CV-B5 $(n=12)$ and CV-B6 $(n=5)$. The clinical and demographic details of all CV-B strains were summarized in Table 1.

\section{Phylogenetic analysis}

A phylogenetic tree was constructed based on partial (479 bp) VP1 gene sequences of CV-B strains $(n=24)$ of the present study to determine the phylogenetic clustering among isolates and to analyze the genetic diversity and molecular aspects of CV-B epidemiology. The VP1 sequence of CV-A1 was included in the phylogenetic tree as an out group. All isolates clustered into six distinct CV-B serotypes (CV-B1 to CV-B6) with their respective prototype strains (Fig. 1). The genetic relationships of the study strains within and between CV-B serotypes were further analyzed.

The analysis revealed that three Pakistan (PAK) strains, detected in 2013 from Balochistan (BN) and Khyber Pakhtunkhwa (KP) Provinces were clustered into the CV-B1 and showed $19 \cdot 2 \%(17 \cdot 2-20 \cdot 9 \%)$ mean nucleotide divergence with their reference strain (accession no. M16560). In addition, two genetic clusters were identified in this serotype: one contained two study strains (PAK-MEV3757B and -3688B) from BN, and the other contained the one strain (PAK-MEV5726A) from KP. The nucleotide divergence of PAKMEV5726A strain with PAK-MEV3757B and PAKMEV3688B strains were $15 \cdot 7 \%$ and $12 \cdot 7 \%$, respectively. The closest relative strain of PAK-MEV5726A was an Indian strain A210 (accession no. JX513569) having $6.7 \%$ nucleotide divergence. CV-B2 serotype contained only one study strain (PAK-MEV2058A) from Sindh (SD) province and had $16.4 \%$ nucleotide divergence with its prototype strain (Ohio-1; accession no. AF081312). Similarly, two strains (PAK-MEV4565A 
Table 1. Clinical and demographic data of acute flaccid paralytic patients with $C V$-B infection characterized in this study

\begin{tabular}{lllllll}
\hline \hline $\begin{array}{l}\text { Serotype identified } \\
\text { in this study }\end{array}$ & Isolate ID & Sex & $\begin{array}{l}\text { Age } \\
\text { (months) }\end{array}$ & Fever & $\begin{array}{l}\text { Asymmetric } \\
\text { paralysis }\end{array}$ & Progression \\
\hline CV-B1 & PAK-MEV3757B & $\mathrm{F}$ & 30 & Yes & Yes & Yes \\
CV-B1 & PAK-MEV3688B & M & 86 & No & Yes & Yes \\
CV-B1 & PAK-MEV5726A & M & 21 & Yes & Yes & Yes \\
CV-B2 & PAK-MEV2058A & F & 24 & Yes & Yes & Yes \\
CV-B3 & PAK-MEV1452A & F & 36 & Yes & Yes & Yes \\
CV-B3 & PAK-MEV4565A & M & 24 & Yes & Yes & Yes \\
CV-B4 & PAK-MEV076B & M & 36 & Yes & Yes & Yes \\
CV-B5 & PAK-MEV1620B & M & 4 & Yes & Yes & Yes \\
CV-B5 & PAK-MEV1177A & M & 16 & Yes & Yes & Yes \\
CV-B5 & PAK-MEV467B & M & 18 & Yes & Yes & Yes \\
CV-B5 & PAK-MEV5777A & F & 36 & Yes & Yes & Yes \\
CV-B5 & PAK-MEV5959A & M & 36 & No & Yes & Yes \\
CV-B5 & PAK-MEV3586B & F & 23 & No & Yes & Yes \\
CV-B5 & PAK-MEV5926A & M & 49 & Yes & Yes & Yes \\
CV-B5 & PAK-MEV3271A & M & 4 & Yes & Yes & Yes \\
CV-B5 & PAK-MEV5959A & M & 36 & No & Yes & Yes \\
CV-B5 & PAK-MEV2005B & M & 23 & Yes & Yes & Yes \\
CV-B5 & PAK-MEV3903A & F & 72 & Yes & Yes & Yes \\
CV-B5 & PAK-MEV5810B & M & 36 & Yes & Yes & Yes \\
CV-B6 & PAK-MEV674B & F & 18 & Yes & Yes & Yes \\
CV-B6 & PAK-MEV2475A & M & 24 & Yes & Yes & Yes \\
CV-B6 & PAK-MEV4600A & F & 18 & Yes & Yes & yes \\
CV-B6 & PAK-MEV627A & M & 18 & Yes & Yes & Yes \\
CV-B6 & PAK-MEV4553A & F & 36 & Yes & Yes & Yes \\
\hline \hline
\end{tabular}

M, male; F, female; CV, coxsackievirus.

and PAK-MEV1452A) isolated from Sindh clustered in CV-B3 and showed $21 \cdot 3 \%$ nucleotide divergence with each other. In addition, CV-B4 serotype had only one study strain (PAK-MEV076B) isolated from KP province and their closest relatives were the strains (PK1Pak92, accession no. AF160019 and P234Pak92, accession no. AF160018) earlier isolated from Karachi, Pakistan in 1992 having 11.2\% nucleotide divergence. Furthermore, phylogenetic analysis revealed the existence of two genetic clusters (I-II) in CV-B5 serotype. Each cluster differed with each other by at least $11 \cdot 2 \%(11 \cdot 2-14 \cdot 2 \%)$ mean nucleotide divergence. Cluster I contained three strains (PAK-MEV1620B, $-1177 \mathrm{~A},-467 \mathrm{~B})$ isolated from KP and Punjab (PB) patients, while cluster II contained nine strains (PAK-MEV5777A, -5659B, -3586B, -5926A, -3271A, $-5959 \mathrm{~A},-2005 \mathrm{~B},-3903 \mathrm{~A}$ and $-5810 \mathrm{~B})$ from PB, SD and BN isolated in 2013. The closest relative of both clusters was strain (accession no. GU323568) isolated from Sweden and had mean nucleotide divergence $9 \cdot 2 \%(9 \cdot 0-9 \cdot 7 \%)$ and $9 \cdot 18 \%(8 \cdot 2-11 \cdot 9 \%)$, respectively. CV-B6 serotype is composed of five study strains collected from KP and SD provinces having 20.4\% $(20 \cdot 1-20 \cdot 9 \%)$ mean nucleotide divergence with their reference strain (Schmitt, accession no. AF081313). The closest relative of the CV-B6 study strains was isolate N-165 (accession no. JN203635) isolated from Indian patient having $15 \cdot 2 \%(12 \cdot 7-16 \cdot 4 \%)$ mean nucleotide divergence.

\section{Genetic divergence among CV-B study isolates}

Six distinct CV-B serotypes could be individualized and appear more clearly in Figure 1. Pairwise sequence comparisons among CV-B study isolates are summarized in Table 2. Isolate of CV-B2 serotype was found the most divergent study strain and differed in nucleotide sequence from other CV-B isolates by $20 \cdot 1$ to $31 \cdot 3 \%(11 \cdot 4-20 \cdot 5 \%$ amino acid sequence difference) while it showed $11 \cdot 2-18 \cdot 4 \% \quad(2 \cdot 0-4 \cdot 0 \%$ amino acid) nucleotide divergence within the serotype. The more study isolates $(n=12)$ were classified into CV-B5 serotype. The strains within CV-B5 differed from one another by up to mean $8 \cdot 0 \%(3 \cdot 0-14 \cdot 2 \%)$ 


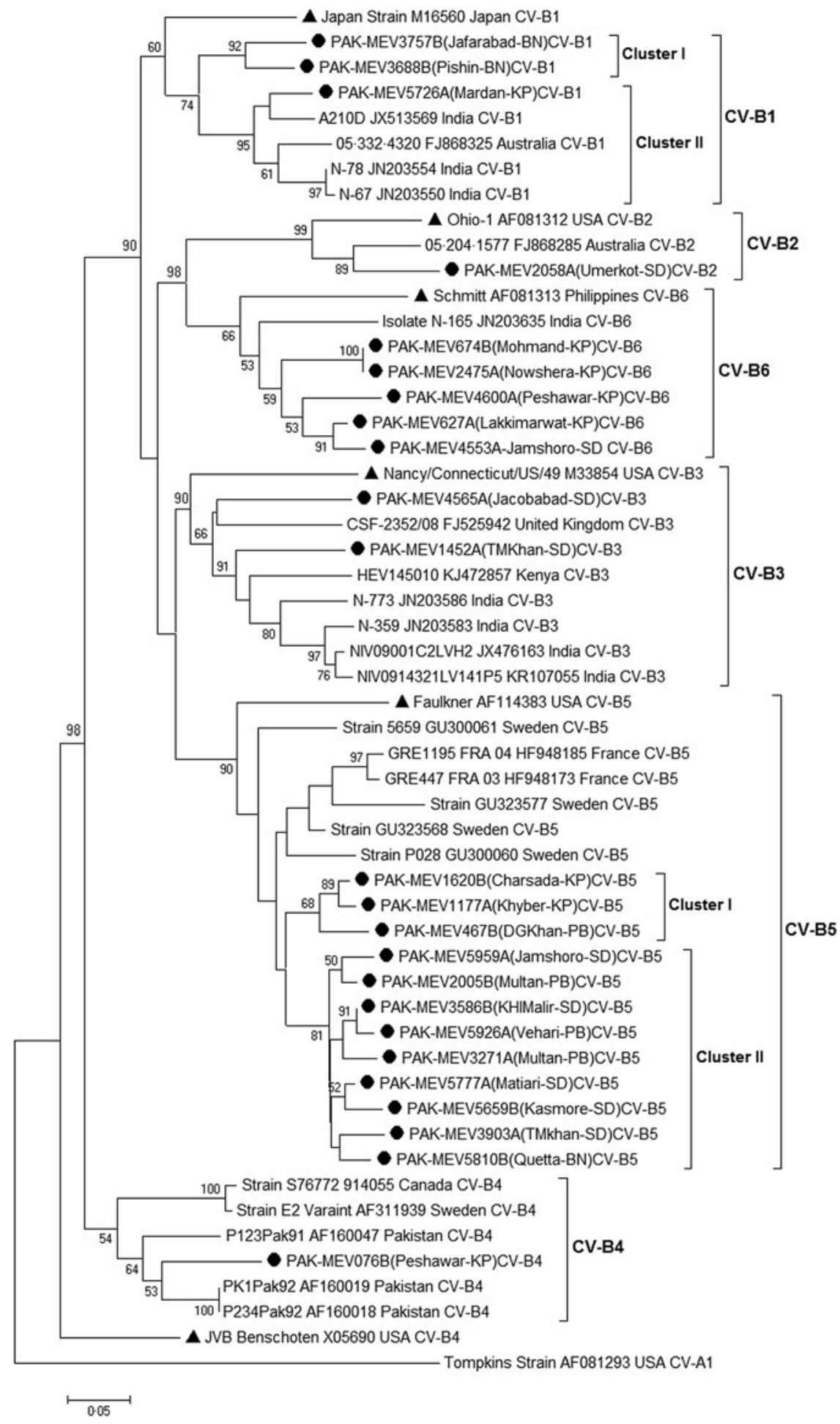

Fig. 1. A Phylogenetic tree based on partial VP1 coding sequence of 24 coxsackievirus B strains including CV-B1 to B6 and representative sequences of coxsackievirus serotypes retrieved from GenBank (accession numbers are included in the virus names). Neighbor-joining (NJ) method in MEGA 5.0 was used and evaluated with 1000 bootstrap pseudo-replicates. Bootstrap values greater than 50 are indicated at the respective nodes and the scale bar represents the evolutionary distance. Clusters are marked on the tree. The isolates from this study and prototype strains are represented by ' $\boldsymbol{}$ ' and ' $\Delta$ ' taxon markers, respectively. 
Table 2. Summary of pairwise nucleotide and amino acid sequence comparisons among coxsackievirus B isolates

\begin{tabular}{|c|c|c|c|c|c|c|c|c|c|c|}
\hline \multirow[b]{2}{*}{ Group } & \multicolumn{5}{|c|}{ Nucleotide (\% divergence) } & \multicolumn{5}{|c|}{ Amino acid (\% divergence) } \\
\hline & $C V-B 1$ & $C V-B 2$ & $C V-B 3$ & $C V-B 4$ & $C V-B 5$ & $C V-B 1$ & $C V-B 2$ & $C V-B 3$ & $C V-B 4$ & $C V-B 5$ \\
\hline \multicolumn{11}{|l|}{ CV-B1 } \\
\hline CV-B2 & $36 \cdot 4$ & & & & & $13 \cdot 1$ & & & & \\
\hline CV-B3 & $31 \cdot 3$ & $42 \cdot 9$ & & & & $11 \cdot 0$ & $20 \cdot 7$ & & & \\
\hline CV-B4 & $29 \cdot 7$ & $47 \cdot 5$ & $38 \cdot 6$ & & & $9 \cdot 1$ & $11 \cdot 1$ & $19 \cdot 2$ & & \\
\hline CV-B5 & $32 \cdot 1$ & $41 \cdot 1$ & $29 \cdot 2$ & $35 \cdot 9$ & & $6 \cdot 5$ & $15 \cdot 8$ & $11 \cdot 1$ & $16 \cdot 5$ & \\
\hline CV-B6 & $36 \cdot 3$ & $38 \cdot 4$ & $35 \cdot 2$ & $39 \cdot 8$ & $33 \cdot 7$ & $12 \cdot 1$ & $17 \cdot 0$ & $18 \cdot 2$ & $15 \cdot 5$ & $19 \cdot 3$ \\
\hline
\end{tabular}

nucleotide sequence divergence (up to $0 \cdot 3 \%$ amino acid sequence difference). Whereas within CV-B1, CV-B3 and CV-B6 serotypes, nucleotide sequences varied by up to mean $12 \cdot 2 \%(8 \cdot 2-15 \cdot 7 \%), 18 \cdot 7 \%$ and $9 \cdot 9 \%(0-13 \cdot 4 \%)$, respectively (up to $0 \cdot 4 \%, 9 \cdot 1 \%$, $2 \cdot 7 \%$ amino acid sequence difference, respectively).

\section{VP1 deduced amino acid sequence analysis}

A comparison of the deduced amino acid sequences of the BC-loop regions of the study strains with their respective reference prototype strains was conducted to investigate the possible amino acid changes in the region. The BC-loop is generally located on the viral surface (VP1) and is associated with enteroviral infectious properties. Therefore, it has been suggested that unique sequence variations in the BC-loop might result in conformational changes that can alter antigenicity in these viruses. Figure 2 shows the alignment of 30 amino acids in the VP1 gene corresponding with amino acid $74-113$ of the VP1 from all strains studied in this work. An analysis of the amino acid sequences of the BC-loop region (amino acid 84-93) of all strains showed that the strains of CV-B2 and CV-B4 had no amino acid change, while strains belonging to CV-B1, CV-B3, CV-B5 and CV-B6 had some changes in this region. All study strains from CV-B1 had serine (S) to threonine (T) substitution at position 86 (S86T), while CV-B3 strains had substitution of the amino acid, alanine (A) to serine (S) at position 87 (A87S). Similarly, the substitution of the 88th and 89th amino acids; aspartic acid (D) to asparagine (N) (D88N) for CV-B5 and lysine (K) to threonine (T) (K89T) for CV-B6 strains were found, respectively.

\section{DISCUSSION}

The Global Polio Eradication Initiative (GPEI) has succeeded in reducing the number of polio cases worldwide, but NPEVs (Echoviruses, CV and enterovirus $68,70,71,77$ ) have been reported frequently in association with AFP worldwide [18-21]. Similar to other NPEVs, the neurological complications of CV infections include AFP, encephalitis, meningoencephalitis and aseptic meningitis [22-24]. CV-B destroy motor neurons leading to the paralysis [25]. Though, mouse models for $\mathrm{CV}$ infection of the central nervous system are available, but much remains to be learned to understand the pathogenesis of this disease [26, 27]. Most research on CV has focused on infection of heart and pancreas. In Pakistan, the role of CVs in causing paralysis is not very clear due to lack of enterovirus surveillance system in the country. The CVs reported earlier in the country were identified in the context of AFP surveillance because poliovirus is the pathogen of interest and detailed testing for identification is not required for NPEVs. Additionally, to the best of our knowledge there is no study on CV-B serotypes in Pakistan that explored their molecular epidemiology, genetic diversity and possible link with AFP. Therefore, in the current study, all these questions were addressed.

The coxsackievirus B serotypes (CV-B1 to B6) have been identified for more than 50 years causing a wide spectrum of diseases and are repeatedly represented in listings of the most commonly isolated enteroviruses [28]. Their genetic analysis has improved our understanding of evolution, epidemiology and pathogenesis $[29,30]$. In the current study, all six serotypes of CVs were identified with high degree of genetic diversity, which is particularly important for a country with no enterovirus surveillance system and a relatively short observation period, i.e. 1 year. Our study also identified distinct grouping pattern of CV-B1 and CV-B5. The phylogenetic analysis of CV-B5 and CV-B6 strains that had circulated in different provinces of the country showed that they can be divided into two clusters. The nucleotide divergences between 


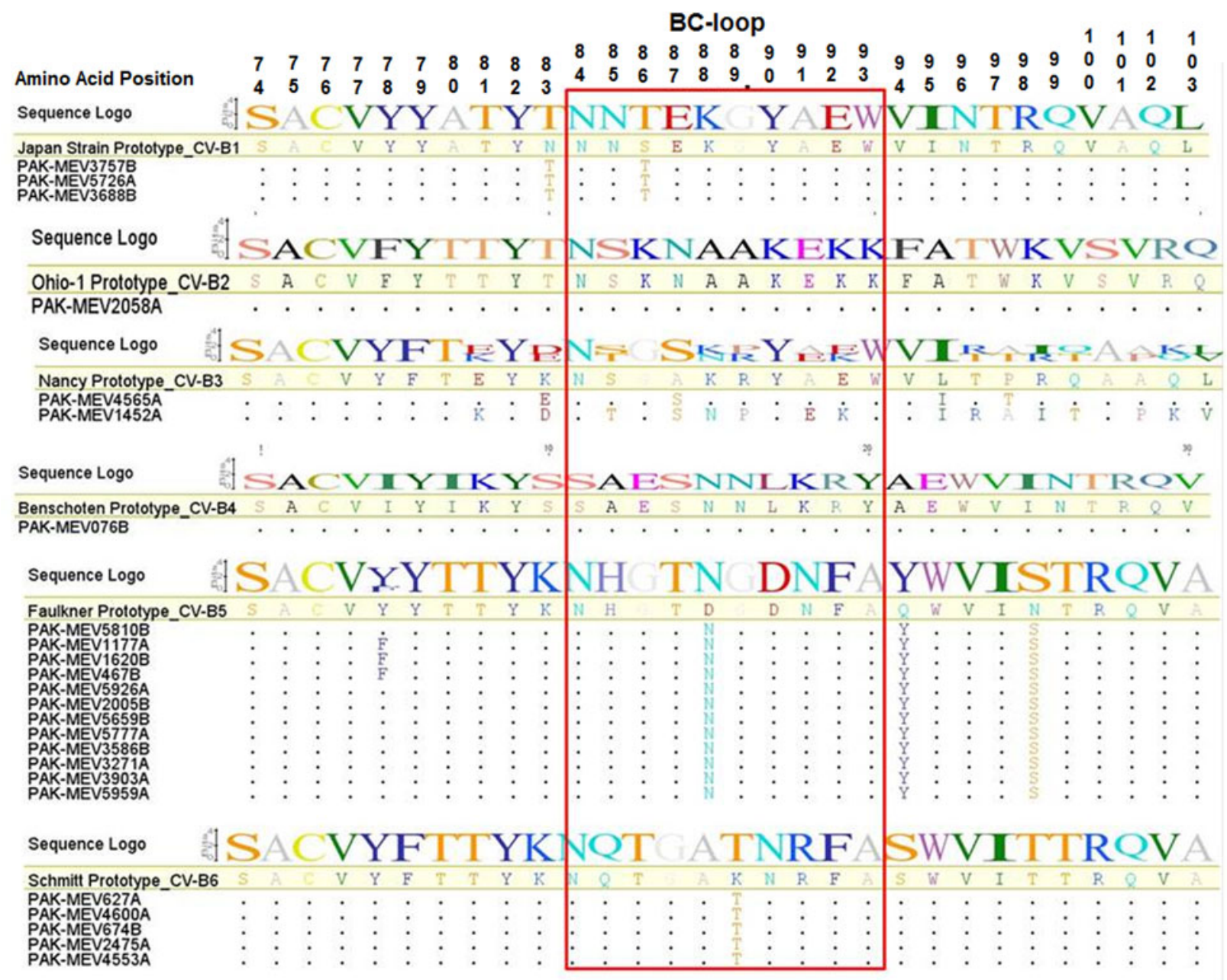

Fig. 2. Alignment of deduced amino acid sequences of the VP1 polyprotein of CV-B1 to B6 strains with their respective prototype sequence showing only the BC Loop with flanking regions. The sequences within the rectangle show variable sites within the BC-loop region. Strain designations are on the left-hand side of the alignment. Dots (.) represent amino acids identical with respect to CV-B1 to B6 prototype strain and the amino acid symbols indicate where unrelated amino acids exist with reference to prototype strain. In sequence logo the height of the logo at each site is equal to the total information at that site and the height of each symbol in the logo is proportional to its contribution to the information content.

study strains and the corresponding virus serotype strains reported in other countries were relatively high. In addition, the presence of multiple distinct genetic clusters is very important observation because the emergence of new genetic clusters of enterovirus in a particular area is often associated with large-scale outbreaks [31]. Furthermore, temporal and geographical comparisons of the study strains with previously characterized CV-B were limited by the availability of comparison sequences in the country. Till to date, there are only three CV-B4 nucleotide sequences in GenBank from Pakistan under accession numbers AF160018, AF160019 and AF160047. Therefore, the information on the range of CV-B serotypes circulating in Pakistan and their nucleotide sequences from the present study contribute significantly to the database of circulating enterovirus strains. These baseline data can be used in the future for determining temporal and geographical relationships of CV-B viruses, which is particularly useful for outbreak investigations and for detecting the emergence of new genetic clusters of enteroviruses.

The BC-loop is one of the regions associated with viral antigenicity, and substitutions resulting in conformational changes in this region are believed to play a role in host adaptation of picornaviruses [32]. In this study, the data analysis of deduced amino acid sequences in BC-loop region in the VP1 gene revealed four major substitutions at positions 86,87 , 88 and 89 in strains, on comparison with the 
representative prototype strains from four different serotypes of coxsackieviruses (CV-B1, CV-B3, CV-B5 and CV-B6), respectively (Fig. 2). It is possible that these changes in the $\mathrm{BC}$ loop region might have played some role in conformational changes and associated with viral antigenicity. However, our findings do not confirm this hypothesis, but more detailed investigations by sequencing of all capsid proteins and conformational analyses may give the better answer.

This study found a different detection frequency pattern of CV-B serotypes. Overall CV-B5 (50\%) strains were isolated more as compared with other serotypes of CV-B. These findings are in line with data reported earlier as CV-B5 consistently appears in the top five commonly identified enterovirus types in the USA and France [5, 33] and has exhibited the highest annual prevalence in a number of countries. Similarly, it has been found an emerging serotype that correlated with some outbreaks of meningitis and other diseases in the last 10 years [34]. In our study, the low frequency of other CV-B types might indicate low circulation of these strains in Pakistani population. It is important to note that the sensitivity of RD cells for CV-B is reported to be low in different reports, but the present study was able to isolate and detect at least one representative of each of the six CV-B serotypes using the RD cell. However, it is possible that some CV-B strains that cannot be propagated in RD and may have been undetected. Therefore, additional cell lines (e.g., MRC-5, Vero, RMK, BGMK and A-549) are recommended for isolation of such viruses in culture to elucidate their actual patterns of circulation.

There are many NPEVs that have been reported as the probable etiologic agents of paralytic illness clinically resembling poliomyelitis, including CV-B [35]. In earlier studies, five coxsackievirus B serotypes (CV-B2 to B6) have been repeatedly isolated from the stool of clinical paralytic poliomyelitis patients with slight to moderate paralysis and all these patients had no evidence of poliovirus infection [36]. CV-B2 and CV-B5 were isolated from stool of patients having spinal paralytic illness in which poliovirus was excluded [37] while CV-B2 to B4 were recovered from brain and spinal cord of patients with spinal paralytic disease [35]. In another study conducted in South Africa, Yui and Gledhil isolated CV-B from the stool of patients presenting with limb paralysis and hospitalized in Kalafong Hospital [38]. CV-B1, B2, B4 and B5 were also isolated from stool of AFP patients in Hong Kong during 2012 [39]. Similarly, in our study, the isolation of CV-B serotypes from AFP patients demonstrated their possible association with paralysis. Additionally, in all these patients, at the time of onset of disease, fever, asymmetrical paralysis and rapid progression were found (Table 1). Most patients had residual weakness on 60 days follow up investigation. Consequently, all these observations propose $\mathrm{CV}-\mathrm{B}$ as the possible causative agent of paralysis. Furthermore, the different animal studies exhibit their neurotropic properties in experimentally infected animals that provide convincing evidence that these viruses have the capacity to produce neurological disease, which may clinically simulate poliomyelitis [27].

This data reveal the isolation of diverse CV-B from wide geographic regions of the country from the stool of paralytic patients. As we are approaching toward global eradication of poliomyelitis, consideration toward NPEV infections causing AFP is an equal cause of concern. Therefore, there is need to monitor such viruses by either the polio eradication program or the larger state health care system for better characterization and epidemiological investigation.

\section{ACKNOWLEDGEMENTS}

The authors gratefully acknowledge World Health Organization AFP surveillance staff working in Pakistan for their technical support. This study was conducted without any kind of external funding, grant or financial support. All work was conducted at Department of Virology, National Institute of Health, Chak Shahzad, Park Road, Islamabad-45500, Pakistan.

\section{DECLARATION OF INTEREST}

None.

\section{REFERENCES}

1. Tracy S, Oberste MS, Drescher KM. Group $B$ Coxsackieviruses. Heidelberg: Springer Berlin Heidelberg, 2008.

2. Dalldorf G, Sickles GM. An unidentified, filtrable agent isolated from the feces of children with paralysis. Science 1948; 108(2794): 61-62.

3. Mark AP, Raymond PR. Enteroviruses: Polioviruses, Coxsackieviruses, Echoviruses, and Newer Enteroviruses, 5th edn. Philadelphia: Lippioncott Williams \& Wilkonson, 2001, pp. 839-894.

4. Knowles NJ, et al. In King $A M Q$, Picornaviridae: In: Virus Taxonomy: Classification and Nomenclature of Viruses: Ninth Report of the International Committee 
on Taxonomy of Viruses. King AMQ, et al. eds. Elsevier, 2012, pp. 855-880.

5. Khetsuriani N, et al. Enterovirus surveillance - United States, 1970-2005. MMWR Surveill Summ 2006; 55 (8): 1-20.

6. Pallansch MA, Oberste MS. Enteroviruses and parechoviruses. In: Specter SC, et al. eds. Clinical Virology Manual. ASM Press, 2009, pp. 249-282.

7. Hamada Y, Ono MDY, Tsubosaki H. A case of coxsackie virus B-1 infection with poliomyelitis-like paralysis. Abstracts of Acta Paediatrica Japonica (Domestic Edition) 1963; 67(7): 413-418.

8. Bendinelli M, Friedman H. Coxsackieviruses: A General Update. USA: Springer, 2013.

9. Oberste MS, et al. Molecular evolution of the human enteroviruses: correlation of serotype with VP1 sequence and application to picornavirus classification. Journal of Virology 1999; 73(3): 1941-1948.

10. Mirand A, et al. Prospective identification of HEV-B enteroviruses during the 2005 outbreak. Journal of Medical Virology 2006; 78(12): 1624-1634.

11. WHO. Polio Laboratory Manual, 4th edn. Geneva, Switzerland: World Health Organization (WHO/IVB/ 04·10), 2004

12. Kilpatrick DR, et al. Rapid group-, serotype-, and vaccine strain-specific identification of poliovirus isolates by real-time reverse transcription-PCR using degenerate primers and probes containing deoxyinosine residues. Journal of Clinical Microbiology 2009; 47(6): 1939-1941.

13. Oberste MS, et al. Comparison of classic and molecular approaches for the identification of untypeable enteroviruses. Journal of Clinical Microbiology 2000; 38(3): 1170-1174.

14. Kroneman A, et al. An automated genotyping tool for enteroviruses and noroviruses. Journal of Clinical Virology: The Official Publication of the Pan American Society for Clinical Virology 2011; 51(2): 121-125.

15. Tamura K, et al. MEGA5: molecular evolutionary genetics analysis using maximum likelihood, evolutionary distance, and maximum parsimony methods. Molecular Biology and Evolution 2011; 28(10): 2731-2739.

16. Schneider TD, Stephens RM. Sequence logos: a new way to display consensus sequences. Nucleic Acids Research 1990; 18(20): 6097-6100.

17. Kearse M, et al. Geneious basic: an integrated and extendable desktop software platform for the organization and analysis of sequence data. Bioinformatics 2012; 28(12): 1647-1649.

18. Dhole TN, et al. Non-polio enteroviruses in acute flaccid paralysis children of India: vital assessment before polio eradication. Journal of Paediatrics and Child Health 2009; 45(7-8): 409-413.

19. Kapoor A, Ayyagari A, Dhole TN. Non-polio enteroviruses in acute flaccid paralysis. Indian Journal of Pediatrics 2001; 68(10): 927-929.

20. Saeed M, et al. Epidemiology and clinical findings associated with enteroviral acute flaccid paralysis in Pakistan. BMC Infectious Diseases 2007; 7: 6.

21. Angez M, et al. Identification of new genotype of echovirus 19 from children with acute flaccid paralysis in Pakistan. Scientific Reports 2015; 5: 17456.

22. Racaniello V. Neurotropic picornaviruses. In: Reiss CS, ed. Neurotropic Viral Infections, 2nd edn. Cambridge: Cambridge University Press, 2008, pp. 3-25.

23. Rhoades RE, et al. Enterovirus infections of the central nervous system. Virology 2011; 411(2): 288-305.

24. Kamei S, et al. Coxsackie B antigen in the central nervous system of a patient with fatal acute encephalitis: immunohistochemical studies of formalin-fixed paraffin-embedded tissue. Acta Neuropathologica 1990; 80(2): 216-221.

25. Hyypia T, et al. Pathogenetic differences between coxsackie $\mathrm{A}$ and $\mathrm{B}$ virus infections in newborn mice. Virus Research 1993; 27(1): 71-78.

26. Feuer R, et al. Coxsackievirus B3 and the neonatal CNS: the roles of stem cells, developing neurons, and apoptosis in infection, viral dissemination, and disease. The American Journal of Pathology 2003; 163(4): 1379-1393.

27. Harvala $\mathbf{H}$, et al. Tissue tropism of recombinant coxsackieviruses in an adult mouse model. The Journal of General Virology 2005; 86(Pt 7): 1897-1907.

28. Romero JR. Pediatric group B coxsackievirus infections. Current Topics in Microbiology and Immunology 2008; 323: 223-239.

29. Dalldorf G. The coxsackie viruses. Annual Review of Microbiology 1955; 9: 277-296.

30. Oberste MS. Comparative genomics of the coxsackie B viruses and related enteroviruses. In: Tracy $S$, Oberste MS, Drescher KM, eds. Group B Coxsackieviruses. Berlin, Heidelberg: Springer, 2008, pp. 33-47.

31. Acton QA. Enterovirus: New Insights for the Healthcare Professional: 2012 Edition: ScholarlyBrief. Atlanta, Georgia: ScholarlyEditions, 2012.

32. Lee ST, Ki CS, Lee NY. Molecular characterization of enteroviruses isolated from patients with aseptic meningitis in Korea, 2005. Archives of Virology 2007; 152(5): 963-970.

33. Antona D, et al. Surveillance of enteroviruses in France, 2000-2004. European Journal of Clinical Microbiology \& Infectious Diseases: Official Publication of the European Society of Clinical Microbiology 2007; 26(6): 403-412.

34. Liu N, et al. An outbreak of aseptic meningitis caused by a distinct lineage of coxsackievirus B5 in China. International Journal of Infectious Diseases: IJID: Official Publication of the International Society for Infectious Diseases 2014; 23: 101-104.

35. Magoffin RL, Lennette EH. Nonpolioviruses and paralytic disease. California Medicine 1962; 97: 1-7.

36. Rezig D, et al. Update on molecular characterization of coxsackievirus B5 strains. Journal of Medical Virology 2011; 83(7): 1247-1254.

37. Rose HM. Viral Infections of Infancy and Childhood: A Symposium of the Section on Microbiology, the New York Academy of Medicine. New York: Hoeber, 1960.

38. Ah Yui L, Gledhill RF. Limb paralysis as a manifestation of coxsackie B virus infection. Developmental Medicine \& Child Neurology 1991; 33(5): 427-438.

39. Chan DY. Fifteen years of acute flaccid paralysis surveillance in Hong Kong: findings from 1997 to 2011. Journal of Paediatrics and Child Health 2014; 50(7): 545-552. 